\title{
Ajuste escolar, clima escolar y apoyo social en bachilleres
}

\section{School adjustment, school climate and social support in high school students}

\author{
Pérez Pulido, Ignacio ${ }^{1, *}$; Zamora Betancourt, María del Rosario ${ }^{1}$; Caldera Montes, Juan Fran- \\ cisco $^{1}$; Reynoso González, Oscar Ulises ${ }^{1}$; Cadena García, Alexa ${ }^{1}$ y Mora García, Olga ${ }^{1}$
}

\begin{abstract}
Resumen:
El trabajo tuvo como objetivos identificar la relación entre el ajuste escolar, el clima escolar y el apoyo social en bachilleres, comparar dichas variables conforme al sexo de los participantes y reconocer la fuerza de las dimensiones del clima escolar y del apoyo social en la predicción del ajuste escolar. La muestra estuvo conformada por 967 estudiantes. Los instrumentos utilizados fueron la Escala Multidimensional Breve de Ajuste Escolar (EBAE-10), la Escala de Clima Escolar (CES) y la Escala de Apoyo Social Familiar y de Amigos (AFA-R). Se detectaron diferencias significativas por sexo $\mathrm{y}$ correlaciones bajas entre las variables estudiadas. Mediante un análisis de regresión logística binomial se generó un modelo que contempla las dimensiones del clima escolar, el apoyo social y el sexo en la predicción del ajuste escolar, siendo el apoyo de amigos el predictor de mayor peso. Se constata la relevancia de las variables estudiadas para lograr un adecuado ajuste escolar y se recomienda la promoción de acciones para incrementar el apoyo social y mejorar los ambientes de estudio en las comunidades escolares.
\end{abstract}

Palabras Clave: Ajuste escolar, Apoyo social, Clima escolar, Rendimiento académico, Estudiantes de bachillerato.

\begin{abstract}
:
The objectives of this work were to identify the relationship between school adjustment, school climate and social support in high school students, compare these variables according to the sex of the participants and recognize the strength of the dimensions of the school climate and social support in the prediction of school adjustment. The sample consisted of 967 students. The instruments used were the Brief Scale of School Adjustment (BSAS10), the School Climate Scale (CES) and the Family and Friends Social Support Scale (AFA-R). Significant differences were detected by sex. Low correlations were detected between the study variables. Through a binomial logistic regression analysis, a model was generated that considers the dimensions of the school climate, social support and sex in the prediction of school adjustment, being the support of friends the predictor of greater weight. The relevance of the variables studied to achieve an adequate school adjustment is confirmed and the promotion of actions to increase social support and improve the study environments in school communities are recommended.
\end{abstract}

Keywords: School adjustment, social support, school climate, Academic performance, students.

\footnotetext{
${ }^{1}$ Centro Universitario de Los Altos. Universidad de Guadalajara

*Correspondencia: delrosario.zamora@academicos.udg.mx
} 
La educación es un factor clave para entender el desarrollo individual, social y profesional de las personas (Rodríguez-Fernández, Droguett \& Revuelta, 2012). La misma se despliega en diferentes contextos, siendo el entorno formal o escolarizado especialmente relevante para la comprensión del desarrollo psicosocial de los jóvenes (Moreno, Estévez, Murgui y Musitu, 2009).

Desde esta perspectiva, la escuela no sólo es una institución encargada de implementar los programas de aprendizaje necesarios para tener una educación eficaz, sino que se convierte en un espacio donde el estudiante interactúa con sus pares y con diferentes figuras de autoridad como el profesorado y los directivos (Moral, Sánchez y Villarreal, 2010). Por tanto, las características que conforman dichas interacciones demandan, por parte de los estudiantes, una serie de adaptaciones o ajustes que no en todos los casos se logran y que no en pocas ocasiones, propician malestar para los estudiantes.

Ante ello, las nuevas orientaciones educativas ya no se enfocan únicamente en el déficit académico, sino que adicionan aspectos sociales y familiares (Hopson, Schiller, \& Lawson, 2014; Kiefer \& Ryan, 2008). Esto hace evidente que el ajuste escolar en estudiantes de preparatoria debe entenderse como un proceso en el que influyen una gran cantidad de factores. Ante esta situación es pertinente retomar la perspectiva teórica del curso de vida, ya que permite visualizar a los estudiantes insertos en un contexto históricosocial que los influye, pero a la vez reconoce aspectos coyunturales y características individuales que les brindan circunstancias diferentes y particulares ante los mismos sucesos sociales (Blanco, 2011; Elder, 1998; Muñiz, 1997).

Específicamente, el ajuste escolar pue- de definirse como un constructo multidimensional relevante para comprender el desarrollo académico de los estudiantes (AntonioAgirre, Azpiazu, Esnaola y Sarasa, 2015; Azpiazu, Esnaola y Ros, 2014), donde interactúan el rendimiento académico, la capacidad para lograr aprendizajes, la actitud hacia la escuela y los profesores, los hábitos, destrezas, habilidades, actitudes, aspiraciones y las nociones de socialización del alumno que derivan en una adecuada adaptación al contexto escolar (Muela, Balluerka y Torres, 2013; Cartagena, 2008; Azpiazu et al., 2014; Orozco et al., 2016; Barreto y Álvarez, 2017).

Por otro lado, el desajuste escolar se relaciona con actitudes y prácticas negativas hacia el profesorado y hacia la escuela en general, así como con el bajo apoyo percibido por parte del alumno, lo cual incrementa el riesgo de abandono escolar y tiene implicaciones negativas para el óptimo desarrollo personal (Bernaras, Jaureguizar, Soroa \& Sarasa, 2017; Estévez y Jiménez, 2014).

Por ello, si se entiende al ajuste escolar como un conjunto de interacciones entre la escuela, la familia y el alumno, es de esperar que diversos estudios lo relacionen con el rendimiento académico, el clima escolar, el apoyo de la familia, de los pares y del profesorado (Antonio-Agirre et al., 2015).

Aunque en la literatura no existe un consenso generalizado sobre los componentes específicos del ajuste escolar, la relación con los compañeros y docentes, la aplicación en las tareas y en el aula, la motivación para asistir a la escuela y un autoconcepto positivo e integrado como estudiante, son claros indicadores de ajuste escolar (Moral et al., 2010).

Particularmente, resulta importante para la comprensión del ajuste escolar el constructo de clima escolar, ya que hace evidente que mantener relaciones positivas con 
compañeros y profesores permite a los estudiantes un adecuado vínculo con la escuela, lo que se convierte en un factor protector contra conductas negativas y alienta el adecuado desempeño académico (Hopson et al., 2014; Whitlock, 2006)

Por lo mencionado en el párrafo anterior es de esperarse que ambientes disfuncionales propicien más dificultades de integración a la escuela y mayor fracaso escolar (Barreto y Álvarez, 2017), así como, pocas expectativas de éxito académico y problemas interpersonales entre compañeros en términos de victimización $\mathrm{o}$ aislamiento social (Estévez, Musito y Herrero, 2005; Azpiazu et al., 2014). Esto resulta más relevante si se toma en cuenta que la adolescencia constituye una etapa crítica para el desarrollo humano, puesto que es cuando mayor riesgo de desajuste clínico, social y escolar se presenta (Bernaras et al., 2017).

Al estar varias horas en la escuela, el alumnado desarrolla habilidades adaptativas y sociales que se asocian con el ajuste escolar (Muela et al., 2013). Dichas habilidades determinan la capacidad para interactuar con adultos y compañeros, de manera que la relación entre pares y docentes, así como el grado de integración social percibido por las y los estudiantes y el profesorado dentro del aula, definen al clima escolar (Cava y Musito, 2001). De igual manera, éste involucra la cohesión, la comunicación, el estilo de dirección docente y variables familiares y sociodemográficas como la edad, género y origen étnico (Orozco et al., 2016; Barreto y Álvarez, 2017).

Otro elemento a considerar al analizar el clima escolar son las metas sociales de los estudiantes, en este sentido, se ha encontrado que en ciertos contextos el comportamiento agresivo y manipulador es una conducta valorada por los alumnos y en consecuencia pue- de facilitar el ajuste social en la escuela, lo que se refleja en consecuencias negativas en el compromiso y logro académicos. (Kiefer \& Ryan, 2008). En estos casos el estudiante puede lograr una adecuada socialización con sus compañeros, sin embargo, los valores en los que se fundamenta dicha convivencia son contradictorios a los fines académicos y de logro escolar.

Diversos estudios muestran que el clima escolar guarda una relación significativa con la presencia de fenómenos como el bullying y conductas violentas en la escuela, ya que un ambiente escolar negativo se caracteriza por una atmósfera de inseguridad y poco apoyo social (Cava, 2011; Valdés y Martínez, 2014). A su vez, las actitudes negativas por parte del estudiante hacia el profesorado y hacia la misma institución tienen un impacto dañino en el clima social del aula de clases (Moreno et al., 2009).

Otro de los factores que influyen en el ajuste escolar es el denominado apoyo social, al cual Lin (1986) define como el conjunto de aportaciones expresivas, instrumentales y provisionales de carácter real o percibido, brindadas por tres diferentes niveles de relaciones sociales: la comunidad, las redes sociales próximas y las personas de confianza.

El apoyo social permite hacer evidente cómo influyen los agentes externos a la escuela en los aspectos de tipo académico, de esta manera los padres, los vecinos y los amigos se han convertido en variables eficaces para predecir el desempeño y el ajuste escolar (Hopson et al., 2014). En este sentido, se esperaría que cada tipo de relación brinde diferentes apoyos a la persona y que entre más cercanas sean, mayor será el impacto de esos apoyos (Lin, 1986). De esta manera, los padres y amigos se ubican dentro del círculo de personas de confianza y por ende se esperaría que sean ellos quienes tengan mayor influen- 
cia en el bienestar del individuo.

Por ello, no es de extrañar que el apoyo social brindado por la familia y los amigos constituya una fuente de soporte en la escuela que fomenta un mejor desempeño académico (Gutiérrez y Gonçalves, 2013; Gutiérrez, Tomás, Romero, \& Barrica, 2017). Otra figura que puede constituirse en un soporte para los estudiantes son los profesores, en este sentido diversas investigaciones han encontrado que la ayuda de los maestros se asocia a mejores niveles de ajuste escolar y logro académico (Demaray, Malecki, Davidson, Hodgson \& Rebus, 2005; Rosenfeld, Richman \& Bowen, 2000).

Otro elemento a considerar respecto a la variable de apoyo social, es el género de los estudiantes, en este sentido se han encontrado que las mujeres perciben mayor apoyo de sus amigas y amigos cercanos, seguido de maestros, padres, compañeros de clase y el personal de la escuela; mientras que para los hombres la principal fuente de apoyo se encuentra en los maestros y los padres, a quienes les siguen los amigos cercanos, el personal de la escuela y por último los compañeros de clases (Rueger, Malecki, \& Demaray, 2010).

De lo anterior, se desprende que disponer de poco apoyo social ya sea por parte de familiares, amigos, compañeros y profesores puede traducirse en dificultades académicas que incluyen problemas de motivación, atención, aprendizaje y cognición (Muela et al., 2013; Barrios \& Frías, 2016).

Así, el apoyo social, específicamente el apoyo familiar, es un referente esencial para el ajuste psicosocial del adolescente en el contexto escolar (Moreno et al., 2009). Además, los problemas de comunicación en el contexto familiar, se relacionan con problemas escolares y a la vez, repercuten en la salud mental de las y los adolescentes (Estévez et al., 2005). Incluso se ha detectado que el bajo apoyo familiar se asocia con la presencia de conductas violentas en la escuela (Valdés y Martínez, 2014). Por otro lado, el apoyo que brinda la amistad entre los iguales es un factor relevante que se relaciona con un menor riesgo de conducta violenta en el aula (Jiménez, Moreno, Murgui y Musitu, 2008).

Por tanto, dada la enorme importancia que para el estudiante y su desempeño representan tales variables, la presente investigación tuvo como objetivos (1) identificar los niveles de ajuste, clima escolar y apoyo social en una muestra de bachilleres de la región Altos Sur de Jalisco; (2) analizar la relación que guardan dichas variables; (3) identificar posibles diferencias entre hombres y mujeres; y (4) identificar cómo influyen el clima escolar y el apoyo de familiares y amigos en el ajuste escolar. Lo anterior resulta pertinente para comprender mejor las dimensiones psicológicas implicadas en el estudio y por ende, proponer estrategias más adecuadas para la mejora de los resultados educativos de los jóvenes que cusan el nivel medio superior o bachillerato.

\section{Método}

\section{Tipo de investigación}

La investigación fue de corte cuantitativo, con un diseño no experimental y transversal de alcance explicativo (Hernández, Fernández y Baptista, 2014).

\section{Participantes}

La población se conformó por los estudiantes inscritos a un bachillerato de tipo tecnológico, ubicado en la Región Altos Sur del Estado de Jalisco, México. Aunque no es el propósito del estudio, es pertinente esbozar brevemente las características generales de la Educación Media Superior en México y en específico de las escuelas de carácter tecnológico, esto con el fin de que el lector cuente con elementos 
suficientes para comprender el contexto donde se realizó la investigación.

En este sentido, la educación media superior en México es de carácter obligatorio y la edad más común para cursarla es de los 15 a los 18 años (Ramírez et al., 2015). Las escuelas que conforman este nivel educativo pueden presentar grandes diferencias, entre las que destacan: el plan de estudios que imparten (bachillerato general, tecnológico, semiescolarizado), el ser pública o privada y el pertenecer a un sistema federal, estatal o a alguna universidad con autonomía (Ramírez et al., 2015; Solís, 2018), Además, se deben contemplar las diferencias sociodemográficas de cada escuela en específico, como: tamaño y ubicación geográfica (Solís, 2018).

En específico, la escuela en donde se realizó el estudio es de carácter público, pertenece al Subsistema de Educación Media Superior del Estado de Jalisco y tiene una orientación tecnológica. La preparación que reciben los estudiantes es gratuita e incluye una formación propedéutica, así como una carrera técnica (Ramírez et al., 2015). De esta manera tiene un carácter bivalente ya que permite a sus egresados optar por continuar sus estudios en cualquier licenciatura o insertarse en el campo laboral como técnico profesional.

\section{Muestra}

El muestreo utilizado fue de tipo no probabilístico incidental ya que se determinó realizar el levantamiento de la información con los estudiantes presentes el día de la aplicación de los instrumentos, esto con la finalidad de captar al mayor número posible de estudiantes. En total participaron 967 estudiantes, de los cuales 439 refirieron ser hombres (45.4\%) y 528 mujeres $(54.6 \%)$, con un rango de edad de 14 a 20 años $(M=16.67 ; \mathrm{DT}=1.160)$. Los criterios de inclusión establecidos fueron contestar la totalidad de los instrumentos y la firma del consentimiento informado.

\section{Instrumentos}

La Escala Multidimensional Breve de Ajuste Escolar (EBAE-10; Moral et al., 2010). Permite medir el grado en el que el adolescente está integrado a su medio escolar. Los indicadores están ordenados en tres factores, el primero "Rendimiento escolar" aborda el desempeño del estudiante en las actividades escolares (p. ej., "Creo que soy buen estudiante"), el segundo "Interés por asistir a la universidad" describe la intención del estudiante por continuar en la vida académica e ingresar a una institución de educación superior (p. ej., "Estoy interesado/a en asistir a la Universidad"), y el último "Problemas de ajuste a la escuela" detalla el nivel de inadecuación al contexto escolar (p. ej., "Tengo problemas con mis compañeros/as de clase"). Consta de 10 ítems, en formato tipo Likert con un rango de 6 puntos, que va desde "Completamente en desacuerdo" a "Completamente de acuerdo". El rango total de la escala es de 10 a 60 . A mayor puntuación obtenida, mayor ajuste escolar. Con el propósito de identificar frecuencias, en este estudio se establecieron rangos o niveles de ajuste escolar, los cuales fueron: Bajo de 10 a 26, Medio 27 a 42 y Alto 43 a 60. Los coeficientes de fiabilidad mostrados en distintas investigaciones han sido satisfactorios, con alfas de Cronbach de .713 y .816 (Moral, et al., 2010; Villarreal, Sánchez y Musito, 2013). En este estudio se reporta un alfa de .742

Escala de Clima Escolar: Relaciones interpersonales (CES: Moos \& Trickett, 1974; Moos, Moos y Trickett, 1987). Esta escala se encuentra conformada por 30 reactivos que abordan la percepción del alumnado hacia la institución escolar y sus diferentes actores. Para el estudio se utilizó la adaptación a población española realizada por FernándezBallesteros y Sierra (1989). Consta de tres dimensiones: implicación escolar (p. ej., "Los alumnos/as ponen mucho interés en lo que 
hacen”); afiliación (p. ej., “A los alumnos/as les gusta ayudarse unos a otros para hacer sus tareas") y ayuda del profesor (p. ej., "Los profesores/as hacen más de lo que deben para ayudar a los alumnos/as"). La escala se compone por reactivos dicotómicos entre verdadero y falso, donde la afirmación se computa como 2 y la negación como 1 . También con la intención de identificar frecuencias por niveles, se establecieron las jerarquías siguientes: Bajo de 30 a 39, Medio 40 a 49 y Alto 50 a 60. Los índices de fiabilidad reportados en investigaciones previas (K-R 20) para cada factor resultaron adecuados: .577, .603, .542 (Jiménez y Lehalle, 2012). En este estudio se reporta un K-R 20 de .755

Escala de Apoyo Social Familiar y de Amigos (AFA-R: González y Landero, 2014): dicho instrumento permite evaluar el apoyo social percibido en dos dimensiones: "Apoyo familiar" y el "Apoyo amigos". La misma está compuesta por 14 ítems; la primera dimensión evalúa la percepción de apoyo, acompañamiento e interés por parte del núcleo familiar en su conjunto (p. ej., "Cuentas con alguien de tu familia para poder platicar cuando lo necesitas"), y la segunda, aborda de forma similar los aspectos mencionados pero percibidos de las relaciones de amistad (p. ej., "Cuentas con algún amigo(a) que te ayude a resolver algún problema"). Es una escala tipo Likert con cinco alternativas de respuesta, que van de "nunca" a "siempre". El rango de respuestas es de 14 a 70 puntos, donde a mayor puntaje, mayor apoyo social. Nuevamente, con el objetivo de detectar frecuencias por niveles, se establecieron los rangos siguientes: Bajo de 14 a 32, Medio 33 a 51 y Alto 52 a 70. Los índices de fiabilidad reportados en otros estudios resultaron adecuados para cada factor o dimensión: 912 y .876 (González y Landero, 2014). En este estudio se reporta un alfa de .892

\section{Procedimiento}

El primer paso consistió en la realización de una reunión con las autoridades del plantel educativo donde se presentó el proyecto de investigación y se obtuvieron los permisos y autorizaciones correspondientes. Posteriormente se convocó a una reunión con los profesores adscritos al plantel donde se expuso el proyecto y en conjunto con el equipo de investigadores se acordó la estrategia para realizar el trabajo de campo. Dicha estrategia consistió en realizar el levantamiento de la información en los salones de clase con el fin de captar al mayor número posible de estudiantes. Además, se llevó a cabo una sesión de capacitación en donde se explicó a los docentes participantes las características de los instrumentos. Ello con la intención de que estuvieran en condiciones de resolver las dudas que pudieran surgir por parte de los estudiantes al momento de contestar.

Cabe mencionar que el equipo de investigadores estuvo presente durante todo el proceso de recolección de información, esto con el fin de mantener cercanía con los encargados de las aplicaciones y resolver las dudas que surgieron en cada momento.

Los aspectos éticos de la investigación se garantizaron mediante un consentimiento informado en donde se explicó a los participantes los fines y propósitos del estudio, se garantizó el anonimato de los participantes y el uso de la información sólo con fines académicos. En el caso de los menores de edad, este documento fue entregado a sus padres a través de los mecanismos que para ello dispuso la escuela. Además, durante la aplicación de los instrumentos se explicó a los estudiantes el objetivo de la investigación y se reiteró el carácter voluntario de su participación, por lo que en cualquier momento podrían negarse a participar en la investigación . 


\section{Resultados}

En primer término se presentan medias, desviaciones estándar y distribuciones de frecuencias por niveles (bajo, medio y alto) de los puntajes totales de las pruebas aplicadas (Tabla 1).

En la tabla se aprecia que la media de Ajuste escolar es de $48.86(\mathrm{DE}=6.737)$, lo que la ubica dentro del nivel alto de la prueba. Por su parte, el Clima escolar obtuvo un promedio de $47.56(\mathrm{DE}=4.743)$ que corresponde al nivel medio (aunque cercano al alto) y el Apoyo social familiar y de amigos registró una media aritmética de $51.80(\mathrm{DE}=10.501)$ la cual también pertenece al mismo nivel.

Respecto a los porcentajes por rango, destaca que la mayoría de los alumnos se ubican en un rango Alto de Ajuste escolar (84\%). En la escala de Clima escolar el 58\% de los estudiantes se posiciona en el rango medio, mientras que, en el Apoyo social, el rango Alto es el que concentra un mayor porcentaje $(57.7 \%)$.

Posteriormente, para identificar las diferencias entre hombres y mujeres se utilizó la prueba de U de Mann Whitney. En la tabla 2, se reportan los resultados totales de las pruebas y sus respectivos factores; así como su correspondiente tamaño del efecto.

Como se muestra en dicha tabla, en la prueba de Ajuste escolar se encontraron dife- rencias significativas por sexo, siendo las mujeres quienes lo presentan en mayor medida $(Z=-4.382 ; \mathrm{p}=.000)$. En lo que toca a los resultados por factores se encontraron diferencias significativas en el de Problemas de ajuste a la escuela $(Z=4.950 ; p=.000)$, donde los hombres son quienes presentan puntajes más altos. En ambos casos la $r$ de Rosenthal permitió identificar que el tamaño de las diferencias es pequeño (Coe y Merino, 2003).

Por lo que toca al en la prueba de clima escolar se detectaron diferencias significativas en dos de sus factores, el de Implicación $(Z=-2.762 ; p=.006)$ y en el de Ayuda del profesor $(Z=-2.120 ; p=.034)$, siendo en ambos casos las mujeres quienes presentaron mejores puntajes. Al igual que en las los casos anteriores, el tamaño de estas diferencias es pequeño $(\mathrm{r}=0.09$ para el primero y $\mathrm{r}=0.07$ para el segundo).

Por último, en el caso del Apoyo social, si bien no se identificaron diferencias significativas en el total de la prueba, no sucede lo mismo en sus factores, ya que los hombres perciben más apoyo por parte de sus familias $(Z=-2.451 ; p=.014)$, mientras que las mujeres de sus amigas(os) $\quad(Z=4.516$; $\mathrm{p}=.000)$. Por lo que respecta al tamaño de las diferencias, en ambos factores también se encontró que son bajos $(\mathrm{r}=0.08$ para apoyo familiar y $\mathrm{r}=0.15$ para apoyo de amigos).

Tabla 1

Frecuencias, medias y desviaciones estándar de los totales de las pruebas de Ajuste escolar, Clima escolar y Apoyo social familiar y de amigos

\begin{tabular}{lccccc}
\hline \multirow{2}{*}{ Variables } & \multirow{2}{*}{$M$} & \multirow{2}{*}{$D E$} & \multicolumn{3}{c}{$\%$ por rangos } \\
\cline { 5 - 6 } & & & Bajo & Medio & Alto \\
\hline Total Ajuste escolar & 48.86 & 6.737 & $1 \%$ & $15 \%$ & $84 \%$ \\
Total Clima escolar & 51.80 & 10.501 & $4.3 \%$ & $41 \%$ & $57.7 \%$ \\
Total Apoyo social familiar y de amigos & 47.56 & 4.743 & $6 \%$ & $58 \%$ & $35 \%$ \\
\hline
\end{tabular}

Fuente: Elaboración propia 
Tabla 2

Comparación por sexo de las variables y factores de Ajuste escolar, Clima escolar y Apoyo social familiar y de amigos

\begin{tabular}{lccccc}
\hline \multicolumn{1}{c}{ Variables/Factores } & Mujeres & Hombres & $Z$ & $p$ & $r$ \\
\hline Total Ajuste escolar & 49.55 & 48.03 & -4.382 & $\mathbf{. 0 0 0}$ & 0.14 \\
Rendimiento escolar & 13.01 & 12.80 & -1.718 & .086 & 0.06 \\
Interés por asistir a la universidad & 10.57 & 10.37 & -1.945 & .052 & 0.06 \\
Problemas de ajuste a la escuela & 9.03 & 10.14 & -4.950 & $\mathbf{. 0 0 0}$ & 0.16 \\
$\quad$ Total Clima escolar & 499.76 & 465.04 & -1.929 & .054 & 0.06 \\
Implicación & 506.40 & 457.05 & -2.762 & $\mathbf{. 0 0 6}$ & 0.09 \\
Afiliación & 475.41 & 494.33 & -1.060 & .289 & 0.03 \\
Ayuda del profesor & 501.15 & 463.37 & -2.120 & $\mathbf{. 0 3 4}$ & 0.07 \\
$\quad$ Total Apoyo social & 491.01 & 475.57 & -.856 & .392 & 0.03 \\
Factor apoyo familiar & 463.97 & 508.10 & -2.451 & $\mathbf{. 0 1 4}$ & 0.08 \\
Factor apoyo amigos & 520.94 & 439.57 & -4.516 & $\mathbf{. 0 0 0}$ & 0.15 \\
\hline
\end{tabular}

Fuente: Elaboración propia.

Tabla 3

Correlaciones entre Ajuste escolar, Clima escolar y Apoyo social familiar y de amigos

\begin{tabular}{lccccccc}
\hline & TCE & IMP & AFI & AP & TASFA & AF & AA \\
\hline \multicolumn{1}{c}{ Total Ajuste escolar } & $.338^{* *}$ & $.244^{* *}$ & $.252^{*}$ & $.242^{*}$ & $.320^{*}$ & $.290^{* *}$ & $.236^{* *}$ \\
& & & $*$ & $*$ & $*$ & $.253^{*}$ & \\
Rendimiento escolar & $.187^{* *}$ & $.144^{* *}$ & $.090^{*}$ & $.174^{*}$ & $.237^{*}$ & $.253^{*}$ & \\
Interés por asistir a la universidad & $.069^{*}$ & .020 & .055 & $.074 *$ & $.138^{* *}$ & $106^{* *}$ & $.129^{* *}$ \\
Problemas de ajuste a la escuela & $-.343^{* *}$ & $-.244^{* *}$ & $-.285^{* *}$ & $-.217^{* *}$ & $-.263^{* *}$ & $-.222^{* *}$ & $-.208^{* *}$ \\
\hline
\end{tabular}

Fuente: Elaboración propia.

Nota: $\mathrm{TCE}=$ Total Clima Escolar; IMP= Implicación; AFI= Afiliación; AP= Apoyo del profesor; TASFA= Total Apoyo social familiar y amigos; $\mathrm{AF}=$ Apoyo familiar; $\mathrm{AA}=$ Apoyo amigos.

Enseguida, para identificar correlaciones entre las variables psicológicas de estudio se empleó la prueba Rho de Spearman. Los resultados se pueden apreciar en la Tabla 3.

En la tabla se destaca que el total de Ajuste Escolar y sus factores Rendimiento escolar y Problemas de ajuste a la escuela, correlacionaron significativamente con el total y sus factores, de las pruebas de Clima Escolar y de Apoyo Social Familiar y de Amigos. Los únicos casos en los que no se detectaron asociaciones se dieron en el factor Interés por asistir a la universidad y los de Implicación y Afiliación.
Sobresale además, que las correlaciones significativas detectadas son de carácter positivo a excepción de aquellas en donde estuvo implicado el factor de Problemas de ajuste escolar, en donde son negativas.

Finalmente, se llevó a cabo un análisis de regresión logística binomial mediante el método por pasos (Wald), considerando al Ajuste escolar como variable dependiente, y los factores del clima escolar (implicación, afiliación y ayuda del profesor) y del apoyo social (apoyo familiar y apoyo de amigo) como independientes. 
Adicionalmente se agregó la variable "sexo" al modelo considerando la asociación evidenciada en los análisis anteriores. Conviene referir que en el análisis de regresión se clasificaron las puntuaciones del total de "Ajuste escolar" de forma dicotómica, considerando los puntajes menores a la media de la puntuación total $(\mathrm{M}=48.86)$ como "sin ajuste" $(41.8 \%)$, y los mayores a tal valor como "con ajuste" (58.2\%).

Los resultados indicaron que un quinto análisis mostró las mejores características de regresión al incluir todas las variables independientes.
Como se muestra en la tabla 4 , los coeficientes no estandarizados bajo signo positivo indican que la presencia de Ajuste escolar se acentúa cuando los niveles de "Implicación”, “Afiliación”, “Apoyo del profesor", "Apoyo familiar" y "Apoyo amigos" son mayores. Por otra parte, el alcance explicativo se encuentra entre los valores de Cox y Snell y Nagelkerke, es decir, que el modelo explica entre el $13.5 \%$ y el $18.1 \%$ del total de la varianza. Por otra parte, al realizar un pronóstico entre los datos observados y los valores de predicción, el modelo acierta en el $67.3 \%$ de los casos.

Tabla 4

Modelo de regresión logística binomial

\begin{tabular}{lccccc}
\hline \multirow{2}{*}{ Variables/factores } & \multicolumn{2}{c}{ Coeficientes no estanda- } & \multirow{2}{*}{ Wald } & Sig. & Exp(B) \\
\cline { 2 - 3 } & \multicolumn{1}{c}{$\boldsymbol{B}$} & Error est. & & & \\
\hline (Constante) & -7.713 & .814 & 89.695 & .000 & .000 \\
Implicación & .118 & .039 & 9.265 & .009 & 1.109 \\
Afiliación & .104 & .040 & 6.811 & .019 & 1.089 \\
Ayuda del profesor & .085 & .036 & 5.524 & .000 & 1.059 \\
Apoyo familiar & .058 & .011 & 25.738 & .012 & 1.033 \\
Apoyo amigos & .032 & .013 & 6.239 & .000 & 1.774 \\
Sexo & .573 & .146 & 15.349 & .002 & 1.126 \\
Logaritmo de verosimilitud & 1174.427 & & & & \\
$R^{2}$ de Cox y Snell & .135 & & & & \\
$R^{2}$ de Nagelkerke & .181 & & & & \\
Pronóstico & $67.3 \%$ & & & & \\
\hline
\end{tabular}

Fuente: Elaboración propia 


\section{Discusión y Conclusiones}

El presente estudio tuvo como principal objetivo identificar cómo influyen el clima escolar y el apoyo social de familiares y amigos en el ajuste escolar de estudiantes de preparatoria; además de identificar la relación entre variables y las diferencias por género en dichos rubros. Esto con la intención de abonar al conocimiento sobre los factores que influyen en el desempeño académico de los estudiantes de bachillerato.

Es importante mencionar que la escuela donde se realizó el estudio presenta puntajes medios con tendencia a altos en las tres pruebas. Lo que indica que no existen problemas graves en el ajuste escolar, ni en el clima escolar y que los estudiantes cuentan con apoyos adecuados por parte de familiares y amigos. Estos resultados permiten reforzar los planteamientos referentes a que este tipo de variables contribuyen a construir ambientes favorables y al logro de buenos resultados académicos (Martínez, Povedano, Amador y Moreno, 2012).

En torno a las correlaciones entre las variables estudiadas, los resultados obtenidos concuerdan con investigaciones previas que señalan que las variables contextuales, como el apoyo de la familia y amigos guardan relación positiva con el ajuste escolar (Azpiazu et al., 2014; Rueger et al., 2010), especialmente con el apoyo familiar (Fuentes, Alarcón, Gracia, \& García, 2015; Hopson et al., 2014; Rodríguez-Fernández et al., 2012). Referente al apoyo de los amigos, este correlacionó positivamente con el ajuste escolar, lo cual discrepa con los resultados de Gutiérrez y Gonçalves (2013). La interpretación que se puede dar a estos hallazgos es que en las personas y especialmente en los jóvenes, la familia y los amigos juegan un papel fundamental en el equilibrio psicológico de los mismos y por ende, en el desempe- ño que ocurre en uno de los contextos que ocupan gran parte de su tiempo, a saber el escolar.

Destaca en los resultados que la dimensión Problemas de ajuste a la escuela correlacionó de manera negativa con las variables de clima escolar y apoyo social (incluyendo sus dimensiones), lo que refuerza el planteamiento de la importancia de dichos fenómenos con el adecuado ajuste psicológico de los jóvenes. Ello probablemente se explique debido a que los estudiantes que carecen de soportes adecuados en sus redes de apoyo (familia, amigos y profesores) y residen en ambientes poco favorables (en donde existe poca implicación y carencias en sus lazos filiativos con sus pares), son más proclives a presentar problemas de ajuste o integración escolar.

Por lo que toca a las diferencias por género las mujeres manifiestan un mejor ajuste que los hombres tanto en el total de la prueba como en el factor de Problemas de ajuste a la escuela. Estos resultados son similares a los encontrados en diferentes investigaciones (Antonio-Agirre et al., 2015; Martínez et al., 2012). Si bien el tamaño de estas diferencias es bajo, estos resultados permiten afirmar que las mujeres presentan mayor disposición hacia las actividades escolares y que en futuras investigaciones convendría profundizar sobre los factores que generan estas diferencias.

Respecto a la prueba de clima escolar si bien no se encontraron diferencias significativas en el total de la prueba, sí se detectaron en las dimensiones de Implicación y Ayuda del profesor. Estos resultaros son similares a los hallazgos de Orozco et al. (2016), ya que en relación al género, las mujeres son quienes tienen una mayor implicación escolar y cuentan con mayor apoyo por parte de los profesores. Resultaría interesante profundizar sobre las habilidades sociales con las que cuentan las mujeres para establecer relaciones de 
amistad y que fungen como factor que promueve una mejor percepción del clima escolar y sus dimensiones.

Respecto al Apoyo familiar y de amigos sólo se detectaron diferencias significativas en los dos factores que componen la prueba. En este punto destaca que los hombres se sienten más apoyados por la familia y las mujeres por los amigos o amigas, situación similar a lo encontrado por Rueger et al. (2010). Estos resultados plantean que en la región aún se presentan disparidades de género dentro de la familia, que se ven reflejadas en menor apoyo a las mujeres por parte de los padres. Ante esta situación, las estudiantes refuerzan sus aspiraciones académicas en su grupo de amigas y amigos, con quienes es posible que tengan mayor afinidad.

Para finalizar con la comparación por género es importante destacar que los puntajes obtenidos a través de la prueba de $r$ de Rosenthal señalan que el tamaño de las diferencia en todas las variables se ubico en el rango de pequeño. Si a estos resultados se les suma que los alumnos en general se ubican en rangos de medios a altos en las tres pruebas, es posible afirmar que aunque las mujeres presentan mejores puntajes, estas diferencias no representan una situación de riesgo para los varones.

Por último, el modelo de regresión logística binomial permite confirmar que los factores de implicación, afiliación, ayuda del profesor, apoyo familiar, apoyo de amigos y la variable de sexo, permiten explicar entre el $13.5 \%$ y el $18.1 \%$ del total de la varianza del ajuste, siendo el factor de Apoyo de amigos el de mayor peso, seguido del sexo, la implicación, la afiliación, la ayuda del profesor y por último el apoyo familiar. Estos resultados concuerdan de manera parcial con otras investigaciones, que si bien han encontrado que dichas variables permiten predecir el ajuste escolar, sus hallazgos ubican a cada uno con diferentes valores explicativos (Azpiazu et al., 2014; Bernaras et al., 2017; Hopson et al., 2014). Una posible explicación a estos resultados estriba en la posibilidad de que los estudiantes entablen fuertes relaciones de amistad dentro de la escuela, de manera que establecen objetivos comunes que promueve un adecuado ajuste escolar.

Destaca que el sexo se coloca como la segunda variable en el modelo explicativo, siendo las mujeres quienes presentan los niveles más altos de ajuste escolar, situación similar a lo encontrado en investigaciones que detectan que las variables que predicen el ajuste escolar son diferentes entre hombres y mujeres (Bernaras et al., 2017; Rueger et al., 2010). Si estos resultados se relacionan con el hecho de que en la comparación por sexo las mujeres reportan mejores puntuaciones en implicación, ayuda del profesor y apoyo de amigos, se puede inferir que las relaciones sociales que ellas establecen dentro y fuera de la escuela se construyen en torno a fortalecer su desempeño escolar y por ende les permiten un mejor ajuste en la escuela.

Por otro lado, es pertinente mencionar que el apoyo de los padres es el factor de menor peso en el modelo, lo que difiere de otras investigaciones que identifican a los padres como uno de los factores más relevantes para explicar el ajuste escolar (Gutiérrez et al., 2017; Rueger et al., 2010). Los resultados de la presente investigación, se pueden deber a que la mayoría de los estudiantes que participaron en el estudio provienen de familias en las que los padres tienen un menor nivel educativo, lo que puede propiciar desconocimiento del contexto escolar y por ende que los apoyos brindados por los progenitores sean menos efectivos que el resto de los factores. 
Es conveniente señalar que esto no resta importancia a este tipo de apoyo para el ajuste escolar de los estudiantes, por lo que en futuras investigaciones sería conveniente profundizar en este aspecto para lograr una mejor comprensión de este fenómeno.

Entre las posibles limitaciones del estudio se encuentra que los instrumentos fueron autoaplicados, lo cual es proclive a la deseabilidad social de las respuestas. Se sugiere que en futuras investigaciones se utilicé una metodología mixta que permita obtener mayor información del contexto escolar y las variables que en este interactúan. Del mismo modo, sería conveniente profundizar en las variables culturales y sociodemográficas que influyen en el desarrollo del contexto educativo, esto con la finalidad de que los resultados permitan generar pautas de intervención para reforzar los factores asociados a un mayor rendimiento escolar.

\section{Referencias}

Antonio-Agirre, I., Azpiazu, L., Esnaola, I. y Sarasa, M. (2015). Capacidad predictiva del autoconcepto y la inteligencia emocional en el ajuste escolar autopercibido. Bordón 67(4), 9-25. doi:10.13042/Bordon.2015.67401

Azpiazu, L., Esnaola, I. y Ros, I. (2014). Factores contextuales y variables individuales en el ajuste escolar. International Journal of Developmental and Educational Psychology, 6(1), 327-336. Recuperado de: https:/www.redalyc.org/ html/3498/349851790038/

Barreto. F. y Álvarez, J. (2017). Clima escolar y rendimiento académico en estudiantes de preparatoria. International Journal of Good Conscien, 12 (2), 31-44. Recuperado de: http:// www.spentamexico.org/v12-n2/A2.12(2)3144.pdf

Barrios, M. y Frías, M. (2016). Factores que Influyen en el Desarrollo y Rendimiento Escolar de los Jóvenes de Bachillerato. Revista Colombiana de Psicología, 25(1), 63-82. doi:10.15446/ rcp.v25n1.46921

Bernaras, E., Jaureguizar, J., Soroa, M. \& Sarasa, M. (2017). Scholar and Clinical maladjustment and personal adjustment in 12-18 year-old adolescents. Revista de Psicodidáctica, 22(2), 118127. doi:10.1016/j.psicod.2017.05.003

Blanco, M. (2011). El enfoque del curso de vida: orígenes y desarrollo. Revista Latinoamericana de Población, 5(8), 2-31. Recuperado de http:// www.redalyc.org/articulo.oa? $\mathrm{id}=323827304003$

Cartagena, M. (2008). Relación entre autoeficacia en el rendimiento escolar y los hábitos de estudio en el rendimiento académico en alumnos de secundaria. REICE Revista Iberoamericana sobre calidad, eficacia y cambio en educación, 6(3), 59-94. Recuperado de: https:// www.redalyc.org/pdf/551/55160304.pdf

Cava, M. (2011). Familia, profesorado e iguales: claves para el apoyo a las víctimas de acoso escolar. Psychosocial Intervention, 20(2), 183192. doi: 10.5093/in2011v20n2a6

Cava, M. y Musito, G. (2001). Autoestima y percepción del clima escolar en niños con problemas de integración social en el aula. Revista de psicología general y aplicada, 52(2), 297-311. Recuperado de: https://www.uv.es/ lisis/ mjesus/7cava.pdf

Coe, R., y Merino, C. (2003). Magnitud del Efecto: Una guía para investigadores y usuarios. $R e$ vista de Psicología de la PUCP, 21(1), 145177. Recuperado de: http://revistas.pucp.edu.pe/ index.php/psicologia/article/view/3722/3704

Demaray, M. K., Malecki, C. M., Davidson, L. M., Hodgson, K. K., \& Rebus, P. J. (2005). The relationship between social support and student adjustment: A longitudinal analysis. Psychology in the Schools, 42(7), 691-706. doi:10.1002/ pits. 20120.

Estévez, E. y Jiménez, T. I. (2014). Conducta agresiva y ajuste personal y escolar en una muestra de estudiantes adolescentes españoles. Universitas Psychologica, 14(1), 111-124. doi:10.11144/ Javeriana.upsy14-1.caap

Elder, G. H. (1998). The Life Course as Developmental Theory. Child Development, 69(1), 1-12. doi:10.1111/j.1467-8624.1998.tb06128.x

Estévez, E., Musitu, G. y Herrero, J. (2005). El rol de la comunicación familiar y del ajuste escolar en la salud mental del adolescente. Salud Mental, 28(4), 81-89. Recuperado de: http://www.inprfcd.gob.mx/pdf/sm2804/sm280481.pdf

Fernández-Ballesteros, R. y Sierra, B. (1989). Escalas de clima social FES, WES, CIES Y CES. Madrid: TEA.

Fuentes, M., Alarcón, A., Gracia, E., \& García, F. (2015). School adjustment among Spanish ado- 
lescents: influence of parental socialization. Cultura y Educación, 27(1), 1-32. doi:10.1080/11356405.2015.1006847

González, M. y Landero, R. (2014). Propiedades psicométricas de la Escala de Apoyo Social Familiar y de Amigos (AFA-R) en una muestra de estudiantes. Acta de Investigación Psicológica, 4(2), 1469-1480. Recuperado de: http:// www.scielo.org.mx/pdf/aip/v4n2/v4n2a2.pdf

Gutiérrez, M. y Gonçalves, T. (2013). Activos para el desarrollo, ajuste escolar y bienestar subjetivo de los adolescentes. International Journal of Psychology and Psychological Therapy, 13(3), 339-355. Recuperado de: https:// www.ijpsy.com/volumen13/num3/366/ajusteescolar-y-bienestar-en-adolescentes-ES.pdf

Gutiérrez, M., Tomás, J. M., Romero, I., \& Barrica, J. M. (2017). Perceived Social Support, School Engagement and Satisfaction with School. Revista de Psicodidáctica, 22(2), 111-117. doi:10.1016/j.psicoe.2017.05.001

Hernández, R., Fernández, C. y Baptista, M. (2014). Metodología de la investigación. México: McGraw-Hill

Hopson, L. M., Schiller, K. S., \& Lawson, H. A. (2014). Exploring linkages between school climate, behavioral norms, social supports, and academic success. Social Work Research, 38(4), 197-209. doi:10.1093/swr/svu017

Jiménez, T., Moreno, D., Murgui, S. y Musito, G. (2008). Factores psicosociales relacionados con el estatus social del alumno en el aula: el rol de la reputación social, la amistad, la conducta violenta y la relación con el profesor. International Journal of Psychology and Psychological Therapy, 8(2), 227-236. Recuperado de: https:// www.redalyc.org/articulo.oa? $\mathrm{id}=56080208$

Jiménez, T. y Lehalle, H. (2012). La violencia escolar entre iguales en alumnos populares y rechazados. Psychosocial Intervention, 21(1), 77-89. Recuperado de: https://www.redalyc.org/ articulo.oa? id=179824383007

Kiefer, S. M., \& Ryan, A. M. (2008). Striving for Social Dominance Over Peers: The Implications for Academic Adjustment During Early Adolescence. Journal of Educational Psychology, 100 (2), 417-428. doi.org/10.1037/00220663.100 .2 .417

Lin, N. (1986). Conceptualizing Social Support. In Social Support, Life Events, and Depression (pp. 17-30). Orlando: Academic Press. doi:10.1016/b978-0-12-450660-2.50008-2

Martínez, B., Povedano, A., Amador, L. y Moreno, D. (2012). Clima escolar, satisfacción con la vida y victimización en la escuela. Un análisis del efecto moderador del género. Anales de Psicología, 28(3), 875-882. doi:10.6018/ analesps.28.3.156121

Moos, R. \& Trickett, E. (1974). Manual, Classroom Environment Scale: A social climate scale. Palo Alto, California: Consulting Psychologists Press.

Moos, R., Moos, B. y Trickett, E. (1987). Escalas de clima social: familia (FES), trabajo (WES), instituciones penitenciarias (CIES), centro escolar (CES). Madrid: TEA.

Moral, J., Sánchez, J. y Villarreal, M. (2010). Desarrollo de una Escala Multidimensional Breve de Ajuste Escolar. Revista Electrónica de Metodología Aplicada, 15(1), 1-11. Recuperado de: https://www.uv.es/lisis/sosa/ajust-escolar.pdf

Moreno, D., Estévez, E., Murgui, S. y Musitu, G. (2009). Relación entre clima familiar y el clima escolar: el rol de la empatía, la actitud hacia la autoridad y la conducta violenta en la adolescencia. International Journal of Psychology and Psychological Therapy, 9(1), 123-136. Recuperado de: https://www.redalyc.org/articulo.oa? $\mathrm{id}=56012876010$

Muela, A., Balluerka, N. y Torres, B. (2013). Ajuste social y escolar de jóvenes víctimas de maltrato infantil en situación de acogimiento residencial. Anales de Psicología, 29(1), 197-206. doi:10.6018/analesps.29.1.124941

Muñiz, P. (1997). Trayectorias educativas y deserción universitaria en los años ochenta. México: ANUIES.

Orozco, M., Colunga, C., Vázquez, J., Vázquez C., Ángel, M., Johnson, S. \& Bradshaw, C. P. (2016). Characterization of school climate perception in mexican middle school students. Psychology, 7, 1562-1574. doi:10.4236/ psych.2016.713151

Ramírez, R., Benítez, G. S., Weiss, E., Ramírez, R. G., Remedi, E., \& Torres, M. C. (2015). Desafios de la educación media superior. México. D.F: Senado de la República, Instituto Belisario Domínguez. Recuperado de http:// bibliodigitalibd.senado.gob.mx/bitstream/ handle/123456789/2939/ desafios_educacion.pdf? sequence $=1 \&$ is Allowed $=\mathrm{y}$

Rodríguez-Fernández, A., Droguett, L. \& Revuelta, L. (2012). School and personal adjustment in adolescence: the role of academic self-concept and perceived social support. Revista de Psicodidáctica, 17(2), 397-414. doi:10.1387/ Rev.Psicodidact.3002 
Rosenfeld, L. B., Richman, J. M., \& Bowen, G. L. (2000). Social support networks and school outcomes: The centrality of the teacher. Child and Adolescent Social Work Journal, 17(3), 205 - 225. doi:10.1023/A:1007535930286

Rueger, S. Y., Malecki, C. K., \& Demaray, M. K. (2010). Relationship between multiple sources of perceived social support and psychological and academic adjustment in early adolescence: Comparisons across gender. Journal of Youth and Adolescence, 39(1), 47-61. doi:10.1007/ s10964-008-9368-6

Solís, P. (2018). La transición de la secundaria a la educación media superior en México: El difícil camino a la cobertura universal. Perfiles Educativos, 40(159), 66-89. doi:10.22201/ iisue.24486167e.2018.159.58412

Valdés, Á., y Martínez, E. (2014). Relación entre el autoconcepto social, el clima familiar y el clima escolar con el bullying en estudiantes de secundaria. Avances en Psicología Latinoamericana, 32(3), 447-457. Recuperado de: http:// www.scielo.org.co/pdf/apl/v32n3/v32n3a07.pdf

Villarreal, M., Sánchez, J, y Musitu, G. (2013). Análisis psicosocial del consumo de alcohol en adolescentes mexicanos. Universitas Psychologica, 12(3), 857-873. Recuperado de: www.redalyc.org/articulo.oa? $\mathrm{id}=64730275017$

Whitlock, J. L. (2006). Youth perceptions of life at school: Contextual correlates of school connectedness in adolescence. Applied Developmental Science, 10(1), 13-29. doi:10.1207/ s1532480xads1001_2 